\title{
GaAs arrays for X-ray spectroscopy
}

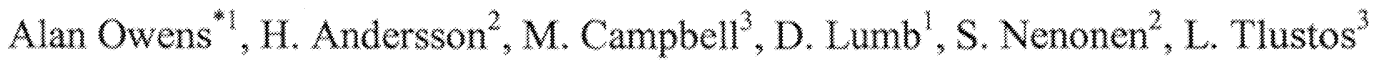 \\ 'Science Payload and Advanced Concepts Office, SCI-AT, ESA/ESTEC, Postbus 299, 2200AG, \\ Noordwijk, The Netherlands \\ ${ }^{2}$ Metorex International Oy, PO Box 85, FIN-02201 Espoo, Finland \\ ${ }^{3}$ CERN, ETT division, 1211 Geneva 23, Switzerland
}

\begin{abstract}
We present results from our compound semiconductor laboratory program and describe the development of a large area GaAs imaging array for planetary remote sensing applications. The device is fabricated from $\sim 150$ micron thick epitaxial material, patterned into a $64 \times 64$ pixel array, back-thinned and contacted. It will be flip-chip bump bonded onto a custom designed, fully spectroscopic, low noise ( $<20 \mathrm{e}-\mathrm{rms}$ ) active pixel sensor ASIC. At present, the ASIC is still under development and so in order to validate and qualify the various technological steps, we have produced a GaAs imager based on the MEDIPIX-1 format using a MEDIPIX-1 readout chip. In X-ray tests, the device was found to work well with a bump yield of $99.9 \%$. After flat field corrections, the spatial uniformity of the array was commensurate with Poisson noise.
\end{abstract}

Keywords: Compound semiconductors, GaAs, X-ray arrays.

\section{INTRODUCTION}

$X$-ray imaging is an essential tool for a wide range of disciplines from astrophysics to material science. Whilst the majority of applications rely on analog integrating formats, the future development of this field lies in the exploitation of spatially resolved spectroscopy and in particular, using materials that can operate at room temperature with near Fano limited energy resolution. Although the bulk of research in direct X-ray imaging has concentrated on $\mathrm{Si}$, GaAs is potentially far superior for the following reasons.

1) Its band-gap energy is sufficiently wide $(1.43 \mathrm{eV})$ to allow room temperature operation, but narrow enough that its Fano limited energy resolution is close to that of Si.

2) Its density is twice that of Si ensuring excellent stopping power well into the hard X-ray region - a particularly attractive property for dental and medical applications.

3) It has a much larger bulk resistivity than Si allowing higher fields to be used for charge collection, which in turn allows thicker devices to be fabricated without compromising efficiency and spectral resolution.

4) Its electron mobilities are about a factor of 6 higher than Si, allowing faster operation.

Additionally, like Si, devices fabricated on semi insulating material can be self-isolating and are therefore ideally suited to integrated circuit fabrication and replication techniques.

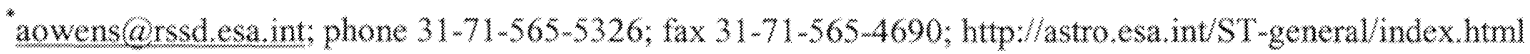

CERN LIBRARIES, GENEVA

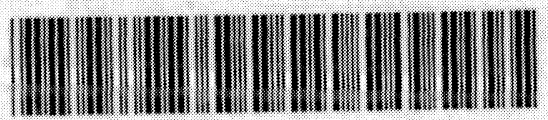

CM-P00050908 
A new generation of high resolution, direct detection GaAs X-ray imagers is being developed by the European Space Agency in cooperation with industry. The program is geared towards X-ray astronomy and remote planetary sensing, but has now been found to have wider applications [1]. In particular, the devices are ideally suited for the imaging of low density, low contrast media. Our research program initially concentrated on monolithic pad detectors and small format arrays. The aim of this work was to address the material, electronic and technological problems that needed to be solved in order to develop mega pixel, spectroscopic imagers with near Fano performances [2]. Building on this work, we have now produced a series of intermediate and large format arrays, intended for planetary remote sensing applications [3]. In addition to the technical effort required to produce spectroscopic grade material and subsequent processing into arrays, considerable effort has also been expended to produce a fully spectroscopic ASIC as well as develop the flip-chip bump bonding techniques to attach it to the sensor.

\section{MATERIAL PRODUCTION}

The imager program has relied heavily on extensive material science research and development. The sensors are fabricated on semi-insulating GaAs wafers using chemical vapour phase deposition (CVPD) techniques. During material processing and growth, the epi-layers and associated structures are subjected to a variety of analytical tests to characterize the material and the technique of producing ultra-pure GaAs layers. For example, Hall effect measurements indicate that impurity concentrations are $<10^{13} \mathrm{~cm}^{-3}$ and the electron mobilities at $77 \mathrm{~K}$ are $2 \times 10^{3} \mathrm{~cm}^{2} \mathrm{~V}^{-1} \mathrm{~s}^{-1}$. These agree well with cyclotron resonance measurements of nonequilibrium electrons in $\mathrm{n}_{\mathrm{O}}$-GaAs layers, which show that the mobilities at $1.8 \mathrm{~K}$ are $3 \times 10^{3} \mathrm{~cm}^{2} \mathrm{~V}^{-1} \mathrm{~s}^{-1}$ and the shallow impurity concentration does not exceed $5 \times 10^{12} \mathrm{~cm}^{-3}$. Based on low temperature Photoluminescence (PL), the density of the As anti-site defect (EL2) is estimated to be of the order of $10^{12} \mathrm{~cm}^{-3}$ (the dominant levels are actually EB4 and EL5) and the room temperature carrier lifetimes of the order of 1 $\mu$ s. This is confirmed by transient microwave absorption (MWA), which yields average carrier lifetimes of $(7.0 \pm 0.5) \mu \mathrm{s}$. The corresponding density of EL2 traps in the substrate material is estimated to be about $3 \times$ $10^{15} \mathrm{~cm}^{-3}$ (which is about one tenth of the values normally obtained for semi-insulating bulk material) and the total impurity concentration $\sim 10^{18} \mathrm{~cm}^{-3}$. Finally, high resolution Glow Discharge Mass Spectrometry (GDMS) measurements has shown that the concentrations of common impurities are typically of the order of a few ppB.

\section{ARRAY DEVELOPMENT}

The material science data was used (and still is) to probe material properties and to assess the electrical and isolation properties of individual pixels. The information gained was fedback into both our material science and theory programs to improve crystal growth, metrology, contacting procedures and detector design. Small format arrays were subsequently produced. In this case, the thrust of the research and development program was to improve processing at the foundry and develop low-noise front-end electronics. In fig. 1 we show the development from small format arrays through to an intermediate format array (1024 pixels). The corresponding improvement in the electronic readout is shown in the lower figures. The arrays are fabricated by growing a GaAs epi-layer on a $400 \mu \mathrm{m}$ thick semi-insulating $n+\mathrm{GaAs}$ substrate. The thickness of the epi layer is chosen to be in the range 40 to $325 \mu \mathrm{m}$ thick. A $\sim 5 \mu \mathrm{m}$ thick $\mathrm{p}+$ layer is then deposited directly onto 

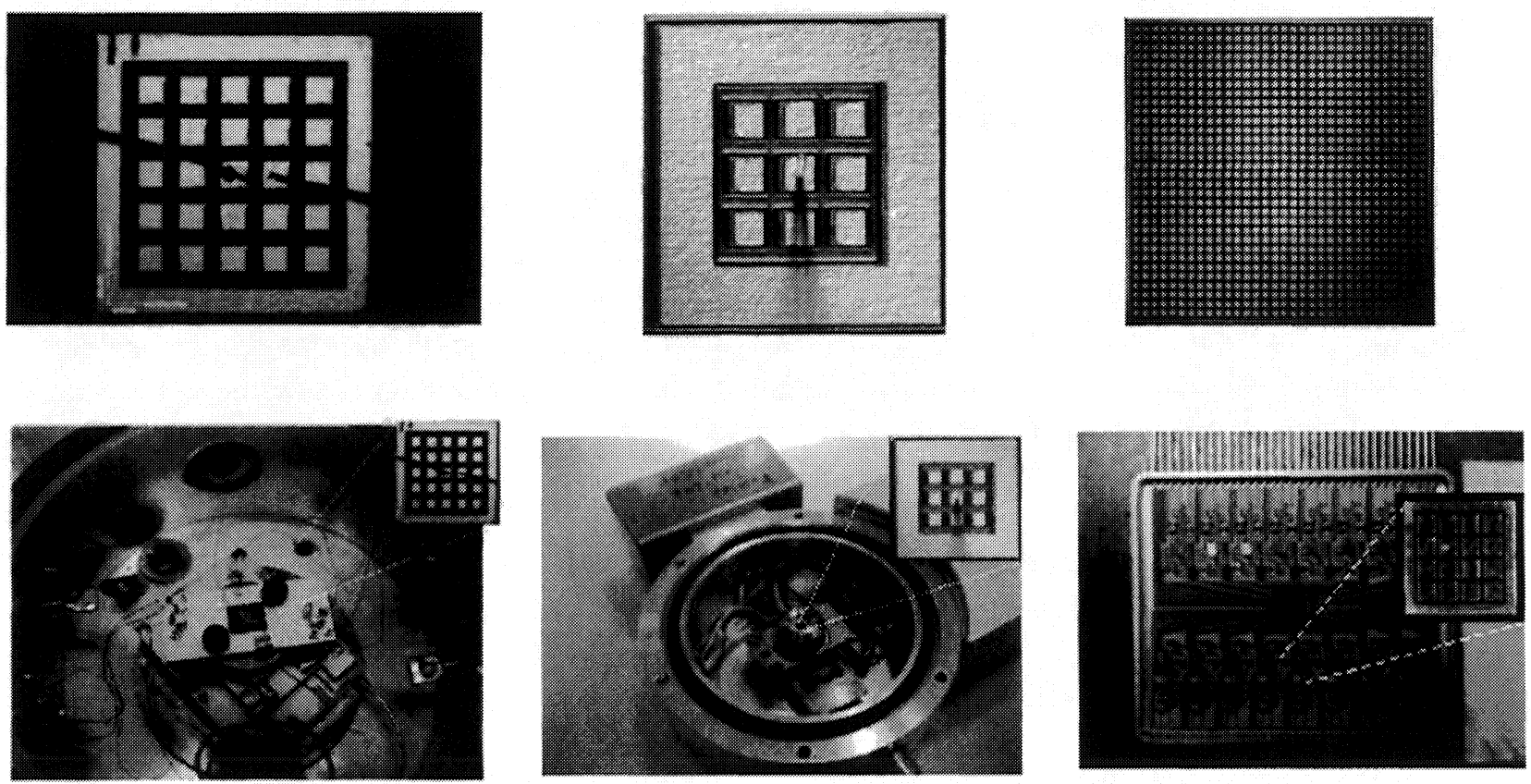

Fig. 1. Top: - Nomarski photomicrographs of small and intermediate format arrays tested at the HASYLAB synchrotron research facility. Below: - the corresponding evolution of the packaging and the front-nd electronics (left to right).

the epi-layer to form a p-i-n structure. This layer is then patterned by etching, to create a pixel structure surrounded by a guard ring (see fig. 1). The guard is $200 \mu \mathrm{m}$ wide and is operated at ground potential. The pixels are metallized with alternate depositions of $\mathrm{Au}, \mathrm{Pt}$ and $\mathrm{Ti}$, forming a Schottky contact, whilst a full ohmic contact $(\mathrm{Ni} / \mathrm{Au} / \mathrm{Ge} / \mathrm{Au})$ is applied to the substrate. The pixel sizes are generally $250 \times 250 \mu \mathrm{m}^{2}$ and the inter-pixel gap, $50 \mu \mathrm{m}$. From the pixel $\mathrm{IN}$ characteristics, the typical current densities at $100 \mathrm{~V}$ are $<0.06$ $\mathrm{nA} / \mathrm{mm}^{2}$ at room temperature. The corresponding pixel leakage current is $0.0024 \mathrm{nA}$ and the inter-pixel resistivities typically $>10^{12} \Omega$.

In fig. 2, we show X-ray test data acquired at the HASYLAB synchrotron research facility for the $32 \times 32$ pixel array shown in the right hand image of fig. 1. Four pixels of the array were instrumented and subjected to rigourous testing on beamline $\mathrm{X}-1$. Leakage currents for this device were typically pA at a bias of $+50 \mathrm{~V}$.
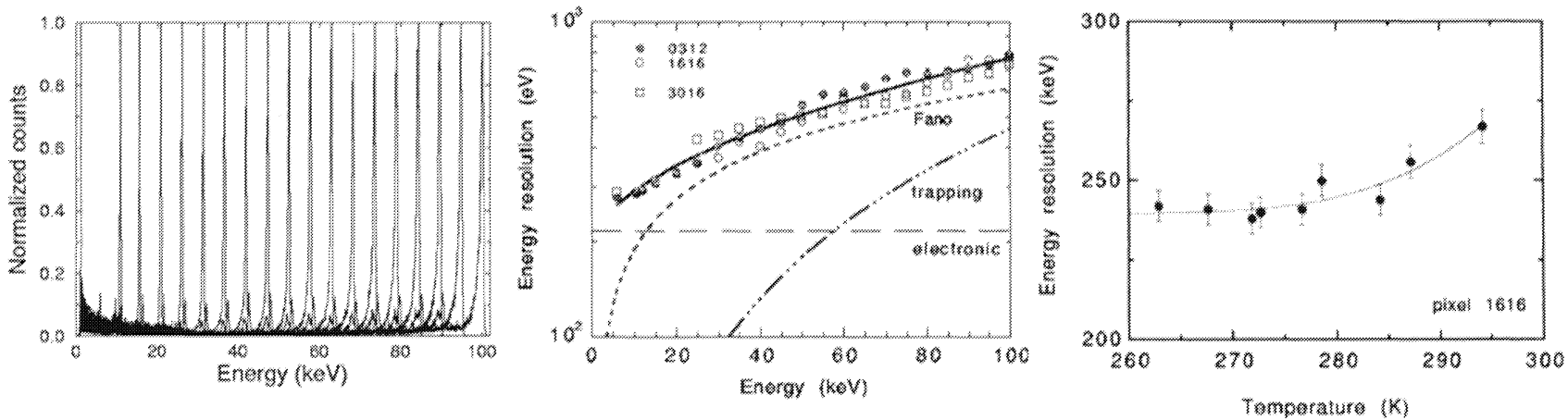

Fig. 2. Composite pulse height spectra (left) and energy resolutions (centre) of monoenergetic lines measured by the 32 $\times 32$ array shown in fig. 1 (right) under pencil beam ilumination at the HASYLAB synchrotron research facility, The right hand image demonstrates that the device can operate at room temperature with little degradation in performance. The incident beam size was $20 \times 20 \mu \mathrm{m}^{2}$. No difference in performance was found under full area illumination. 
In fig. 2 (left), we show a composite of energy-loss spectra recorded by a single pixel over the energy range $10.5 \mathrm{keV}$ to $100 \mathrm{keV}$. Plots for the other pixels are statistically consistent, both in terms of spectral distribution and energy resolution. The smaller peaks which appear at energies of $10.4 \mathrm{keV}$ and $11.9 \mathrm{keV}$ below the photopeaks are due to the Ga and As escape peaks. The energy resolution function for each pixel was determined from its pulse height data and is shown in fig. 2, (center). At room temperature, the resolutions range from $\sim 290 \mathrm{eV}$ FWHM at $5.9 \mathrm{keV}$ to $780 \mathrm{eV}$ FWHM at $100 \mathrm{keV}$. The electronic noise of the system was $\sim 220 \mathrm{eV}$ FWHM. As can be seen, the measured resolutions for all 4 pixels agree within $20 \%$ - the differences almost certainly arise from the difference in stray capacitance of the front-end components. The corresponding resolutions measured under full-area illumination were found to be the same as for pencil beam illumination within statistics, indicating uniform crystallinity and stoichiometry. Likewise, by raster scanning a $15 \mathrm{keV}, 15 \times 15 \mu^{2}$ beam across each of the pixels, the gain uniformity across the pixels (and by implication the entire array) was determined to be statistically flat. These data were verified using a $4 \times 4$ pixel array with all pixels instrumented using purpose designed hybrid electroncs [4] (lower right figure in fig. 1). In this case, all pixels gave identical responses, within errors (to better than the $1 \%$ level), over the energy range 10 to $100 \mathrm{keV}$. Lastly, we note from the plotted energy resolutions at $5.9 \mathrm{keV}$ shown in fig. 2 (right) that, unilike $\mathrm{Si}$, cooling the array below $+10^{\circ} \mathrm{C}$ does not improve its performance.

(a)

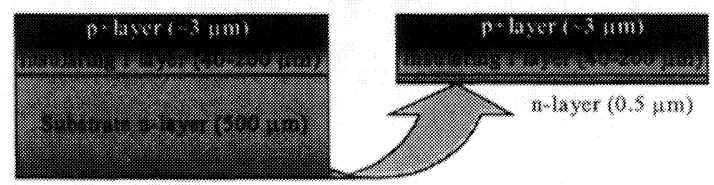

(b)
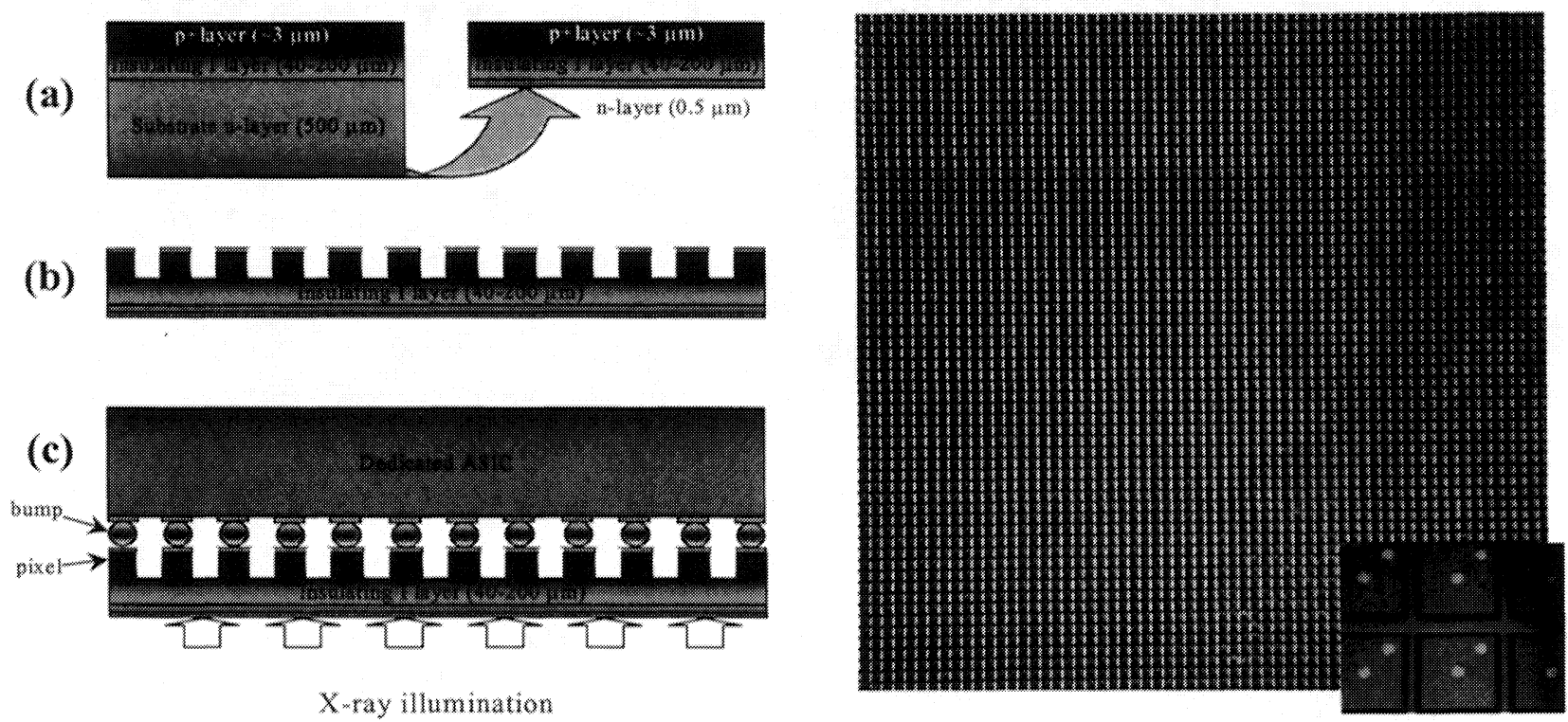

Fig. 3. Left: the process steps required to produce a flip-chip bump bonded array - a) back thinning, b) patterning, etching and contacting and c) flip-chip bump-bonding to the readout ASIC. Right: A $64 \times 64$ pixel GaAs array produced to qualify the bump bonding processes. The inset shows individual pixels in detail. Note, the center hole in the passivation is where the bump will be fixed; the upper hole is used to electrically probe the pixel prior to bumping.

The $\mathrm{p}-\mathrm{i}-\mathrm{n}$ structure was the key to producing near Fano limited performance in both monolithic and small format arrays by drastically reducing leakage currents. This in turn allowed us to test increasingly lower noise preamplifier designs, read-out through the $\mathrm{p}+$ contact and positively biased via the $\mathrm{n}+$ contact to ensure the efficient collection of holes. For array sizes $>6 \times 6$, wire bonding is impractical and so the read-out is only realistically achieved using a custom built ASIC which is connected to the pixelated side of the array by flip-chip, bump bonding. However, this necessitates that the chip is now illuminated through the $\mathrm{n}+$ electrode side, since the ASIC will cover and obscure the pixel side. To avoid excessive X-ray extinction in the nt 

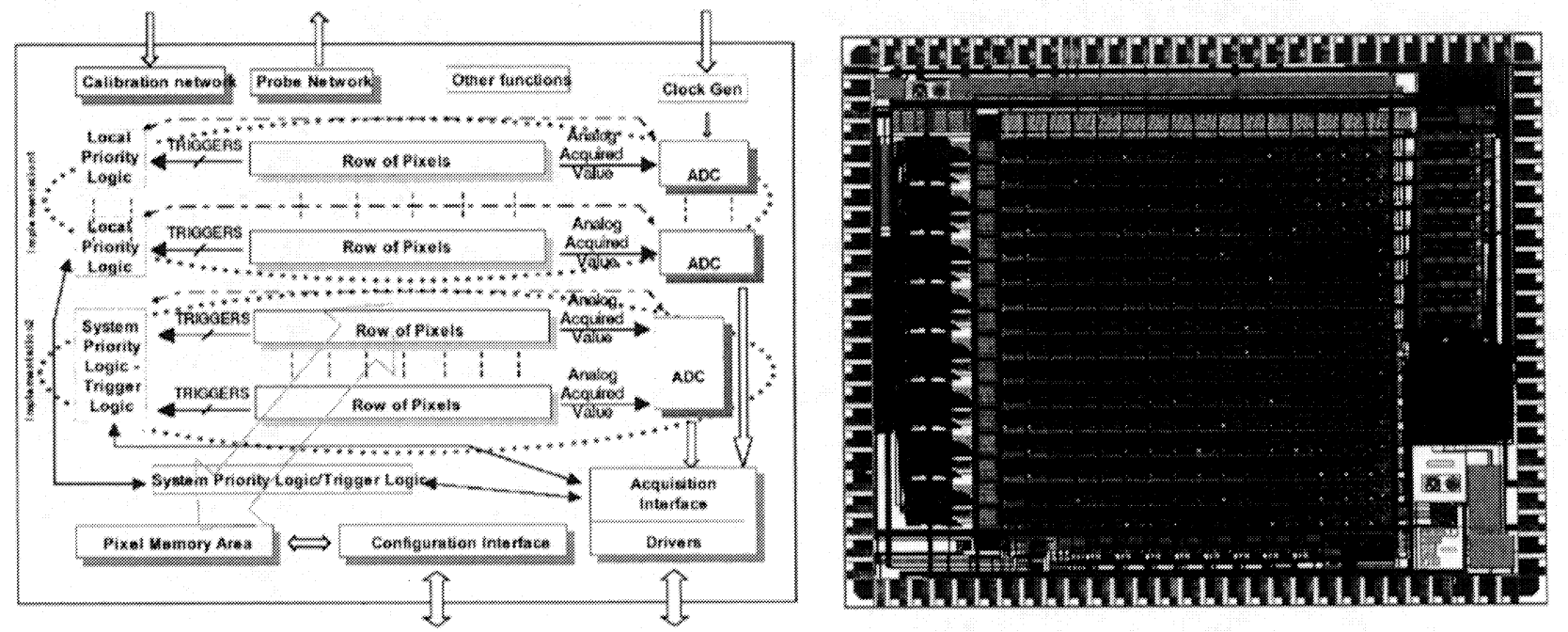

Fig. 4. Left: Block diagram of the ASIC design, based on an active pixel sensor design. Right: the corresponding mask.

layer, this layer has to be substantially thinned or removed. The present experimental goal is to develop a larger array $(64 \times 64$ pixels $)$ in which all pixels are read out with a low noise $(<20 \mathrm{e}-\mathrm{rms})$ spectroscopic ASIC in an APS format. Towards this end, we have begun an ambitious technical program to a) back-thin the substrate, b) develop low temperature bumps c) develop the flip-chip bump-bond process for GaAs, and d) produce the ASIC. Each, of these steps ( $\mathrm{a}, \mathrm{d}, \mathrm{c}$ and d) has been developed in parallel. The essential technological steps in the production of an imager are summarised in fig. 3 (left). A block diagram of the ASIC design is shown in fig. 4 (left) and the corresponding mask in fig. 4 (right). At the present time, the ASIC is still under development - the masks have been delivered to the foundry and the first prototype chips are due for delivery. The thinning was carried out by ORC, Tampere, Finland and flip-chip bump bonding by VTT, Espoo, Finland. Both have proceeded successfully at the component level using rejected GaAs wafers and silicon dummies. Therefore in order to qualify the flip-chip bump-bonding process, and reduce schedule delays due to the late delivery of the ASIC, we decided to bond a representative GaAs chip to an existing (but non-spectroscopic) ASIC - the MEDIPIX-1 chip [5]. Towards this end, we have produced a backthinned GaAs $64 \times 64$ pixel array in the MEDIPIX-1 format (see fig. 3 right). The pixel size is $170 \times 170$ $\mu \mathrm{m}^{2}$ and the thickness of the epi-layer $\sim 150$ microns. Because it was not possible to thin the substrate to an absolute precision of better than a few microns, the substrate was completely removed by mechanical polishing. Tests have shown that the surface uniformity is better than 0.5 microns. The surface was then passivated and an ohmic contact grown directly onto the epi-layer. The chip was then flip-chip bump bonded to the MEDIPIX-1 ASIC. This ASIC is based on the $1 \mu \mathrm{m}$ SACMOS process. Its analog front-end comprises a charge-sensitive pre-amplifier and a shaper. Incoming charge from a semiconductor sensor is amplified and compared with a threshold in a comparator. If the signal exceeds this threshold the event is counted. Fig.5 (left) shows a photomicrograph of the reverse side of the MEDIPIX readout chip prior to bonding with the $\mathrm{SnPb}$ bumps attached. The diameter of the bumps is $25 \mu \mathrm{m}$. In fig. 5 (right) we show the completed flipchipped bump-bonded assembly, ready for testing. As can be seen the process does not appear cosmetic. The completed assembly was then wire-bonded to a MUROS1 interface board [6]. MUROS1 is a multipurpose read-out system based on a PCI standard. Data acquisition is controlled by the Medisoft software [7]. The sensor was operated at room temperature at a bias of $100 \mathrm{~V}$. In fig. 6 (left) we show the first X-ray images of a line pair rule. The rule was laid on top of the chip and the array exposed to an uncollimated ${ }^{109} \mathrm{Cd}$ radioactive source located $\sim 6 \mathrm{~cm}$ above the rule. Because of the large variations in individual pixel thresholds, the image has been flat-fielded using flood illumination from the ${ }^{109} \mathrm{Cd}$ source - the lower energy estimated to be 
around $10 \mathrm{keV}$. Apart from a few dead pixels around the edges of the imager (due to dicing process), only 4 pixels were found to be non-functioning - thus the bump yield is $99.9 \%$. After flat field corrections, the spatial uniformity of the array at $20 \mathrm{keV}$ was commensurate with Poisson noise.
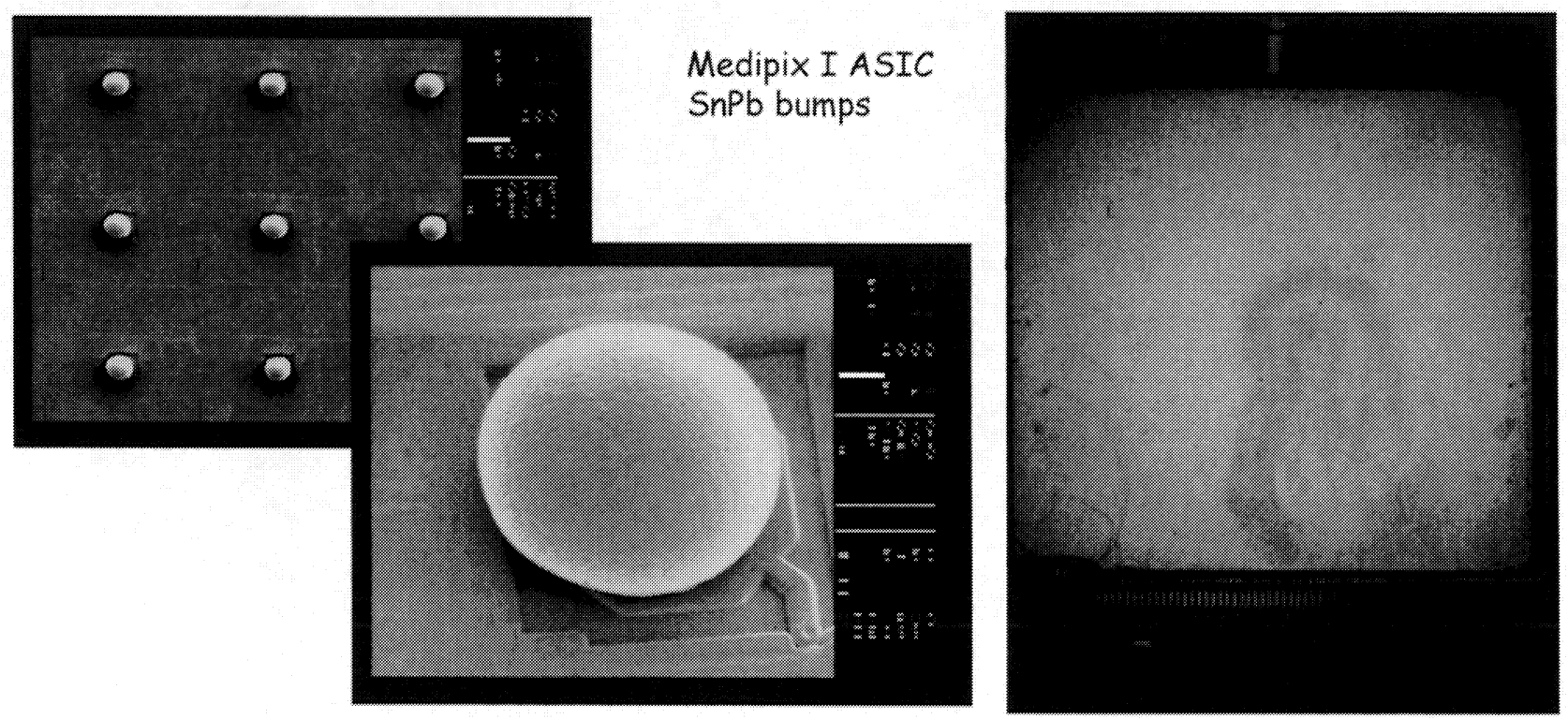

Fig. 5 Left: photomicrograph of some of $25 \mu \mathrm{m}$ diameter bumps attached to the MEDIPIX-1 readout ASIC. Right: the completed flip-chip bump bonded MEDIPIX-1 X-ray imager, as seen from the thinned GaAs side.

Finally, in fig. 6 (right) and fig. 7 we show a pair of images of a "Swatch" and a small sardine, illustrating the imaging quality of the GaAs chip. The data were taken with a conventional X-ray tube using a tungsten target. The objects were mounted on a sample holder and the detector on a precision $x-y$ stage. The detector was then scanned past the object in steps of 0.8 of the detector width using the so-called move and tile method [8]. The resulting composite images have been flat-field corrected and a $3 \times 3$ median filter applied locally around defective pixels.
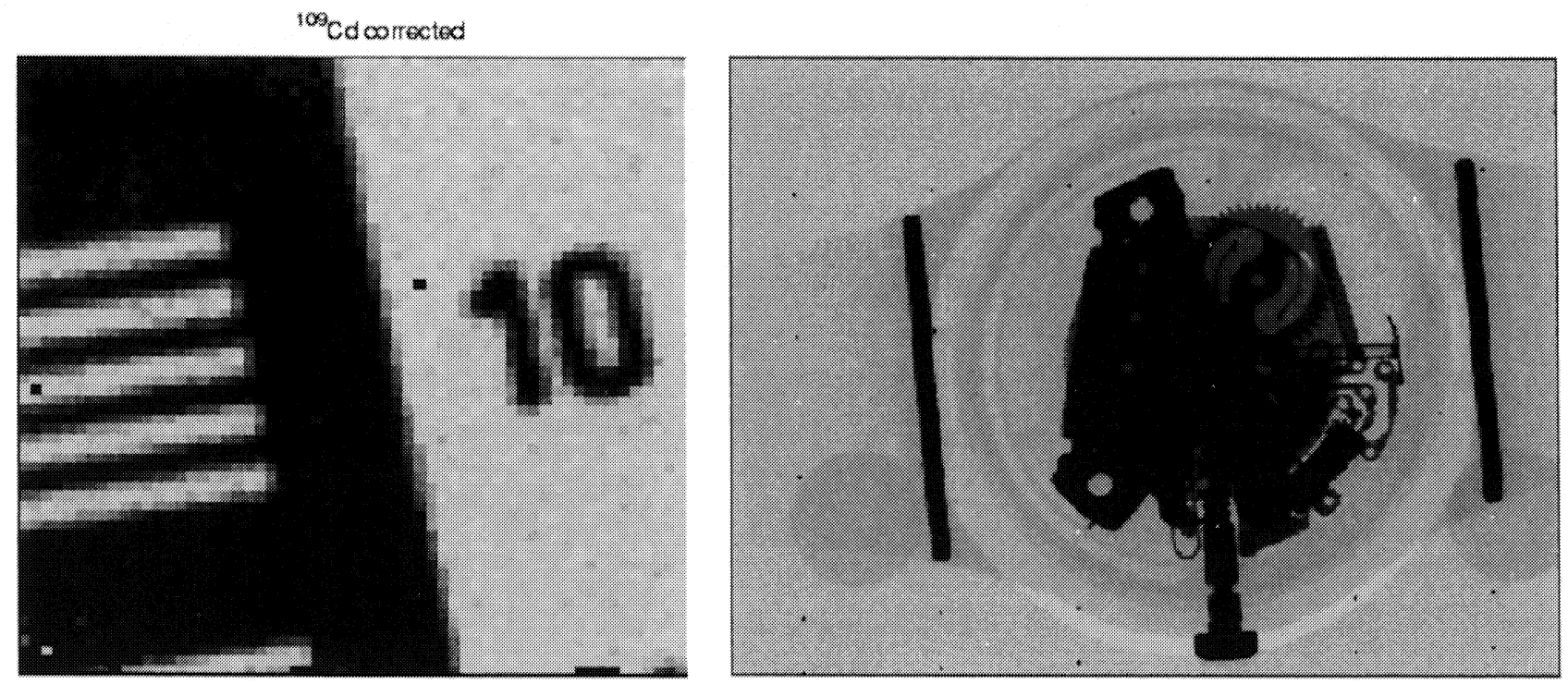

Fig. 6. X-ray image of a line pair rule taken using an un-collimated ${ }^{109} \mathrm{Cd}$ source located $\sim 5 \mathrm{~cm}$ above the target. Right: image of a "Swatch", taken with a conventional X-ray set operating at $55 \mathrm{kV}$ with a W target and a $2.5 \mathrm{~mm}$. Al filter. 

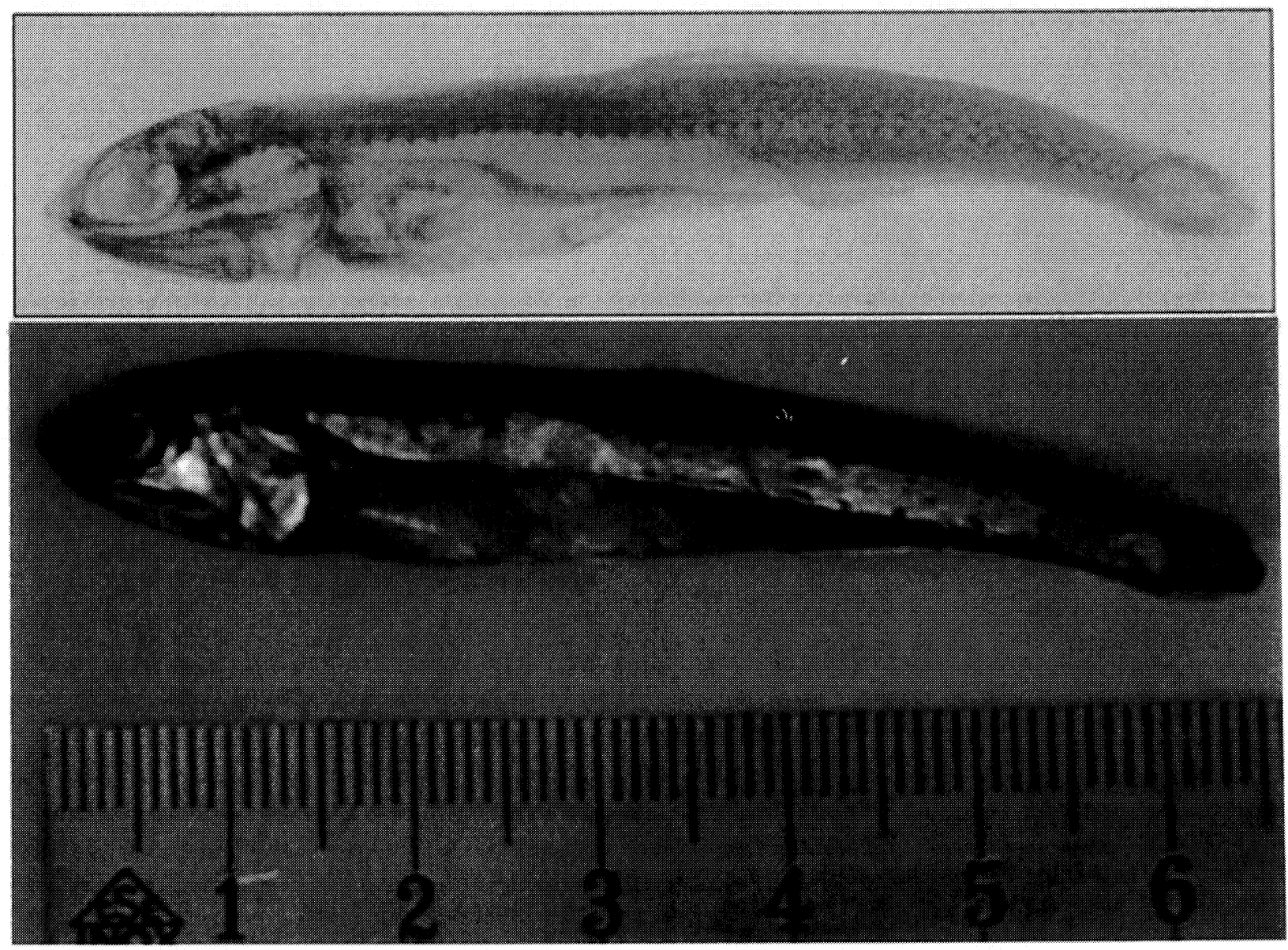

Fig. 7. Top: X-ray image of the sardine, shown in the lower image, taken using an X-ray tube with a tungsten target plus a $2.5 \mathrm{~mm}$ Al fiter. The following tube settings were used $35 \mathrm{kV}$ and $20 \mathrm{mAs}$. The target to object distance was $54 \mathrm{~cm}$.

\section{CONCLUSIONS}

The present results are very encouraging and illustrate the quality of images that can be expected with spatially resolved spectroscopy, especially if coupled with contrast enhancement techniques such as differential X-ray absorbtiometry. Lastly, the fact that GaAs sensors can operate at room temperature has important consequences for a number of applications where power, mass and volume are at a premium $(e, g$., intraoral radiography), alleviating the need for complex and expensive cryogenic systems - as in the case of Ge or Si CCD-type sensors.

\section{REFERENCES}

1. A. Owens, et al, ESA Bulletin number 108 (2001) 32.

2. A. Owens, et al., J. App. Phys., 90 (2002) 5376.

3. A. Owens, et al., Proc. of the SPIE, 4506, (2001) 136.

4. A. Owens, et al., Proc. of the SPIE, 4507 (2001) 42.

5. M. Campbell et al, IEEE Trans. Nucl. Sci. 45 (3) (1998) 751.

6. G. Bardelloni, et al., IEEE Trans. Nucl. Sci., Symp. Med. Imaging Conf., 12 (2000) 57.

7. B. Mikulec, et al., Nucl. Instr. \& Meth., A458 (2001) 352.

8. K. Pfeiffer, et al., Nucl. Instr. \& Meth., A509 (2003) 340. 